Pathologe 2010 · [Suppl 2] 31:183-187

DOI 10.1007/s00292-010-1370-6

Online publiziert: 28. August 2010

(c) Springer-Verlag 2010

\author{
H. Schulze \\ Labor für Pädiatrische Molekularbiologie, Klinik für Allgemeine Pädiatrie, \\ Otto-Heubner Centrum für Kinder- und Jugendmedizin, Berlin
}

\title{
Gegenwärtige Modelle der Thrombozytopoese
}

In einem erwachsenen Menschen zirkulieren etwa $10^{12}$ Thrombozyten. Bei einer durchschnittlichen Lebensdauer von etwa 10 Tagen müssen etwa $1,5-\mathrm{mal}^{10^{11}}$ Thrombozyten täglich ersetzt werden [14, 15]. Darüber hinaus erfordert Blutverlust als Folge von Blutungen eine zusätzliche Nachfrage an Thrombozyten, die jeweils schnell von Vorläuferzellen produziert werden müssen. Obwohl es seit über 100 Jahren bekannt ist, dass Thrombozyten aus zytoplasmatischen Abschnürungen von Megakaryozyten (MKs) entstehen [33], sind die molekular- und zellbiologischen Mechanismen, die diesem Prozess zugrunde liegen, bis heute im Wesentlichen wenig erforscht worden.

\section{Vom Megakaryozyten zum Thrombozyten}

MKs gehören zu den größten Zellen im Knochenmark und können außer durch ihre Größe noch an ihrer Morphologie und dem viellappigen Kern sicher identifiziert werden. Im Vergleich zu diesen statischen Bildern von MKs im Knochenmark, ist unser Wissen von den dynamischen Vorgängen der Abschnürung von Thrombozyten noch sehr limitiert. Dies liegt hauptsächlich daran, dass MKs zu den seltenen Zellen im Knochenmark gehören, wo sie nur etwa 0,05\% aller nukleären Zellen ausmachen.

Thrombopoietin (TPO) wurde 1994 als der wichtigste hämatopoetische Wachstumsfaktor für die Thrombozyten identifiziert, und erst mit der Verfügbarkeit von rekombinantem Protein wurde es relativ einfach, den Prozess der Thrombozytopoese auch ex vivo zu untersuchen [16]. Während der Embryogenese findet die
Hämatopoese in der Leber statt. Aus einer terminiert verpaarten Maus können etwa 15 Feten isoliert und die Leberzellen in vitro zu MKs differenziert werden. Nach 4 bis 5 Tagen bilden sich lange pseudopodienartige zytoplasmatische Ausläufer, die als Proplättchen bezeichnet werden und die reife, funktionale Thrombozyten abschnüren. So konnte in der Tat mittels Zeitraffervideomikroskopie eindrucksvoll gezeigt werden, dass sich Thrombozyten nur an den Enden dieser Proplättchenausläufer bilden können, wo sich tränenförmige Verdickungen mit dem Volumen des abzuschnürenden Thrombozyten bilden [13].

Das derzeitige Modell der Biogenese von Thrombozyten wurde in einigen Übersichtsartikeln aus den letzten Jahren dargestellt [3, 23]. Dieser Artikel beschäftigt sich daher mit einigen Aspekten, die weniger oft beschrieben worden sind:

- Derzeitige Modelle der Thrombozytopoese.

- Wie erfolgt die Bildung von Thrombozyten über die Endothelzellen der Blut-Knochenmark-Schranke hinweg?

- Wie kann ein genaueres Verständnis der zellbiologischen Grundlagen dazu beitragen, im klinischen Alltag Thrombozytopenien und -pathien besser zu erkennen und zu diagnostizieren?

\section{Modelle der Thrombozytopoese}

Als Thrombozytopoese wird der terminale Reifungsprozess bezeichnet, bei dem MKs im Knochenmark mit einer Größe von bis zu $80 \mu \mathrm{m}$ zwischen 100 und 1000 Thrombozyten von 2-3 $\mu \mathrm{m}$ Durchmesser in den Blutstrom abgeben. Alle Modelle, die diesen Vorgang beschreiben, müssen auch einen Schritt beinhalten, der die zelluläre Fragmentation beschreibt. Während der letzen 50 Jahre haben sich hauptsächlich zwei Modelle entwickelt

- zum einen die „Platelet-Territory-

Theorie“, die sich zum gegenwärtigen „Explosionsmodell“ entwickelt hat, sowie

- zum anderen das „Flow Model“, das ursprünglich von Radley u. Haller eingeführt wurde [27] und die Bildung von Proplättchen als unmittelbare Zwischenform bei der Plättchenbiogenese beschreibt [11].

Die von Proplättchen abgeschnürten zytoplasmatischen Fragmente weisen dieselben Eigenschaften (Größe, Ultrastruktur und Reaktivität) auf wie Thrombozyten, die man aus dem peripheren Blut isoliert hat [7]. Da die Mikrotubulifilamente sich nur an der Spitze oder an hantelförmigen T-Endstücken der Proplättchen zu Bündeln aufwickeln, können reife Thrombozyten nur an diesen freien Enden gebildet werden, was dadurch erreicht wird, dass sich die Proplättchen oft verzweigen.

In den letzten Jahren konnte aufgrund dieses Modells gezeigt werden, wie diese Zytoplasmafragmente mit den plättchenspezifischen Granula beladen werden und welche Polarität die einzelnen Mikrotubulifilamente in den Proplättchen und Plättchen aufweisen [24, 28]. Dieses „Proplättchen-Flow-Model“" ist derzeit das favo-

Dieser Artikel basiert auf einem Vortrag, den der Autor auf der 94. Jahrestagung der Deutschen Gesellschaft für Pathologie, Berlin, 2010 gehalten hat. 
risierte Modell der Thrombozytopoese $[6,9,17]$, wobei einige Aspekte - so u. a., dass die in diesem Ansatz erhobenen Daten auf unphysiologischen In-vitro-Experimenten beruhen - von einem „zytoplasmatischen Fragmentationsmodell“" kritisiert werden [18].

Die morphologischen Veränderungen, die ein einzelner sphärischer MK durchgehen muss, um mehrere 100 quasi identische diskoide Thrombozyten zu bilden, sind beeindruckend, da die zellbiologischen und biochemischen Prozesse genau orchestriert werden müssen. Dabei ergeben sich aus zellbiologischer Sicht drei Hauptprobleme der Thrombozytenbiogenese:

1. Bei der Abschnürung von 100 bis 1000 Plättchen aus einer einzelnen Zelle von 50-80 $\mu \mathrm{m}$ wird eine extremer Zuwachs an Oberfläche benötigt, die aus vorher im Inneren des MKs angelegten Membranspeichern gespeist wird. Das aus elektronenmikroskopischen Aufnahmen bekannte ausgebreitete System an inneren Membranen, das mit der äußeren Zellmembran verbunden ist, wurde vor über 5 Jahrzehnten beschrieben [34], aber es ist das Verdienst von Radley in den frühen 198oer Jahren zu erkennen, dass dieses als „Demarkationsmembranen " bezeichnete System nicht die zukünftigen Plättchen voneinander abgrenzt (z. B. in Form von „Platelet-Territories“), sondern dass es besser als Invaginationsmembransystem bezeichnet werden sollte, das als Speicher für die Plättchenbiogenese dient [27]. Allerdings hat sich die biochemische Charakterisierung des Membransystems als schwierig herausgestellt. Wir konnten vor einigen Jahren zeigen, dass während der Ausreifung der MKs das innere Membransystem mit Phosphatidylinositol- $(4,5)$-bisphophat (PIP2) angereichert wird, das kurz vor der Proplättchenbildung in reifen MKs mit Komponenten des Aktin-Zytoskeletts co-lokalisiert [30]. Die Proplättchenschäfte - obwohl aus dem inneren Membransystem hervorgehend, vergleichbar mit einem von innen nach außen gestülpten Strumpf - sind biochemisch von dem
Zellkörper des MKs unterscheidbar. Dieser hat PIP2 verloren und kann mit Annexin $\mathrm{V}$ angefärbt werden. Er wird als letzter Rest des MKs von Makrophagen phagozytiert $[8,30]$. Wie die neugebildeten Aktinfilamente die Membranen nach außen drücken und dabei die Membranasymmetrie aufrechterhalten, bleibt allerdings weiterhin ungeklärt.

2. Die Thrombozyten schnüren sich nicht willkürlich wie Knospen von dem MK ab, sondern werden kontrolliert an den freien Enden der zytoplasmatischen Ausläufer abgegeben. Diese hochverzweigten Proplättchen sind alle mit Mikrotubulifilamenten ausgestattet, die dort - fern vom Zellzentrum - nukleiert werden müssen, allerdings ohne die klassischen Mikrotubuli-organisierenden Zentren (MTOCs). Die einzelnen Filamente weisen sowohl innerhalb der Proplättchen wie auch in den Thrombozyten eine bipolare Orientierung auf [28], und der Nachweis von $\gamma$-Tubulin oder dem Tubulin-bindenden Protein RanBP1o könnte erklären, wie die Filamente gebildet bzw. gebündelt werden $[24,29]$. Allerdings sind wir noch weit von einem konsistenten Verständnis entfernt, inwieweit das Zytoskelett zur Thrombozytenbildung beiträgt.

3. Das vielleicht größte Rätsel bleibt die Frage, wie ein reifer MK, der im Knochenmark an den Sinusoiden lokalisiert ist, seine Thrombozyten über die Endothelzellen der Blut-Knochenmark-Schranke in den Blutstrom abgibt, ohne dabei durch Matrixproteine des Stromas wie Kollagen oder von-Willebrand-Faktor stimuliert zu werden. Dies bleibt den späteren Thrombozyten vorbehalten, wenn sie am verletzten Subendothel durch genau jene Faktoren aktiviert werden $[2,14,21]$. Es ist offensichtlich, dass die In-vitro-Zellkultur diese komplexen In-vivo-Prozesse nicht oder nur unzureichend widerspiegeln kann. Die Bildung von Proplättchen und Thrombozyten ist ein zellautonomer Prozess, dessen letzte Schritte selbst unabhängig von TPO ablaufen $[7,20]$. Allerdings modulieren Proteine der extrazellulären Matrix wie Fibrinogen, Fibronektin, Kollagen Typ I oder Typ IV die Ausbildung von Proplättchen [19]. Das Zusammenspiel von Zell-Zell- und Zell-Stroma-Kontakten, das die Ausreifung von hämatopoetischen Stammzellen zu MKs und weiter zur Abschnürung von Thrombozyten in die Blutbahn reguliert, ist nur unzureichend bekannt.

\section{Wo findet die terminale Thrombopoese statt?}

Unsere Kenntnis der Thrombopoese in vivo beruht im Wesentlichen auf elektronenmikroskopischen Aufnahmen von Knochenmarkschnitten, die zeigen, dass reife MKs in den Verzweigungen der Sinusoiden liegen bzw. zeigen, wie zytoplasmatische Ausläufer von MKs in das Lumen von Gefäßen ragen [21]. Dieser Ansatz, obwohl unverzichtbar, weist einige methodische Probleme auf: Die Präparation hochwertiger elektronenmikroskopischer Aufnahmen ist anspruchsvoll und neigt zu Artefakten. Aufnahmen sind statisch, und es bleibt daher schwierig, daraus dynamische Prozesse abzuleiten.

Im letzten Jahrzehnt konnten einige dieser methodischen Limitationen überwunden werden: zum einen durch den Einsatz transgener Tiere, bei denen spezifische Zelltypen durch fluoreszierende Proteine markiert sind, sowie durch die Weiterentwicklung der Zwei-PhotonenIntravitalmikroskopie. Hierbei erfolgt die Anregung der Fluorophore durch Hochfrequenz-Laser mit der doppelten Wellenlänge, was die Detektion in tieferliegendem Gewebe ermöglicht mit verbessertem Signal-zu-Rausch-Verhältnis.

Unsere Gruppe hat die Plättchenbiogenese in einem Mausmodell untersucht, bei dem ausschließlich MKs und Thrombozyten das gelb-fluoreszierende Protein eYFP exprimieren [30]. Wir konnten zeigen, dass sich die Mehrzahl aller reifen MKs an Sinusoiden und insbesondere an Gefäßgabelungen befinden [14]. Einige Fragmente von MKs konnten auch schon im Knochenmarkstroma identifiziert werden, allerdings waren sie etwa 10- bis 100mal größer als bei den In-vitro-Experimenten. Die durchschnittlichen direkt ins Blut abgebebenen Fragmente wiesen vari- 
able Größen mit einem Durchschnitt von $6 \%$ des Volumens von MKs auf und waren somit etwa 8oo-fach größer als Thrombozyten im peripheren Blut der Maus. Trotzdem unterstützen die Beobachtungen aus diesen Experimenten das „Flow Model“ und sprechen gegen das „Fragmentationsmodell“. Allerdings legen unsere Daten auch nahe, dass zwar die Megakaryozytopoese im Knochenmark erfolgt, dass die terminale Thrombozytopoese aber zumindest teilweise außerhalb des Knochenmarks stattfindet.

Bereits 1893 konnte Aschoff MKs in der Lunge nachweisen [1]. Dieses Konzept wurde in der ersten Hälfte des 20. Jahrhunderts durch zwei Beobachtungen unterstützt:

1. In histologischen Schnitten wurde deutlich, dass MKs durch das venöse Blut in die Lunge transportiert werden und nicht dort reifen und

2. dass die Konzentration von Thrombozyten im Blut vor der Lunge niedriger ist als dahinter [12].

Dies konnte durch Shunt-Experimente an Hunden und Ratten bestätigt wurde [15, 25]. Auch beim Menschen kann man einige MKs im venösen Blut nachweisen, wobei die Konzentration in Gefäßen, die das Knochenmark drainieren (z. B. die V. cava inferior mit $5 \mathrm{MKs} / \mathrm{ml}$ ) höher ist, als in der antekubitalen Vene, die typischerweise für die Blutentnahme verwendet wird. Dies erklärt auch, warum beim Blutausstrich normalerweise keine MKs nachgewiesen werden.

Weil die Thrombozytenkonzentration nach der pulmonalen Passage ansteigt, wird das Kapillarbett der Lunge auch als Gewebefilter bezeichnet, wobei die zugrunde liegende Physiologie rätselhaft bleibt $[15,26]$. Neben einzelnen MKs können auch Plättchenvorläufer im peripheren Blut nachgewiesen werden, was die These unterstützt, dass zumindest nicht alle Thrombozyten bereits als reife Blutbestandteile unmittelbar im Knochenmark freigesetzt werden [4]. In einer aktuellen Studie wurde gezeigt, dass auch reife Thrombozyten in der Peripherie weiter fragmentiert werden können [31], wobei dieser Mechanismus sicherlich nicht maßgeblich zur gesamten Thromboztenproduktion beitragen wird.

Pathologe 2010 · [Suppl 2] 31:183-187 DOI 10.1007/s00292-010-1370-6

(c) Springer-Verlag 2010

\section{H. Schulze}

\section{Gegenwärtige Modelle der Thrombozytopoese}

\section{Zusammenfassung}

Thrombozyten sind korpuskuläre Blutbestandteile, die für die Hämostase essenziell sind. Sie werden mittels zellulärer Fragmentierung von Vorläuferzellen im Knochenmark in den Blutstrom freigesetzt. Obwohl einige Aspekte der Thrombozytopoese gut bekannt sind, konnte der Vorgang in vivo bislang nur unzureichend analysiert werden. Dieser Artikel diskutiert drei aktuelle Aspekte: 1. Es werden Modelle der Thrombozytopoese vorgestellt sowie einige offene Fragen aus Sicht der Zellbiologie. 2. Aktuelle Erkenntnisse, wie Megakaryozyten im Knochenmark über die endotheliale Barriere hinweg Thrombozyten in den Blutstrom abgeben, werden dargestellt. 3. Schließlich wird diskutiert, inwieweit diese Kenntnisse dazu beitragen kön-

\section{Current models of thrombopoiesis}

\section{Abstract}

Platelets are essential corpuscular blood components required for hemostasis. They are released from precursor cells in the bone marrow by cellular fragmentation into the blood stream. Although some aspects of platelet biogenesis are well known, studying its occurrence in vivo has been mostly unsatisfactory. This article reviews some recent findings: It first introduces current models of thrombopoiesis including open questions on cell biology. Second, it presents current findings on the release of megakaryocytes over the endothelial barrier into the blood stream where platelet production takes place outside the bone marrow. Finally, it reports on the translation of the more basic science into an improved understanding of novel para- nen, neue Thrombozytenparameter wie das mittlere Plättchenvolumen (MPV), die Verteilungsweite (PDW), Plättchenkrit (PCT) sowie die immature Plättchenfraktion (IPF) so zu verstehen und anzuwenden, dass sie für den klinischen Alltag informativ werden. Ein genaueres Verständnis dieser Parameter und der Thrombozytopoese kann dazu beitragen, die Krankheitsursache und die beste Therapie zu finden - ein Bereich, in dem bislang die Thrombozytenzahl oft der einzige Parameter ist, um ein Blutungsrisiko abzuschätzen.

Schlüsselwörter

Thrombozyten · Immunthrombozytopenie · Megakaryozyten · Thrombozytopoese . Knochenmark meters from automated blood counters surveying platelet volume (MPV), distribution width (PDW), mass (PCT) and the immature platelet fraction (IPF) into clinics where platelet counts are often the only accessible parameter to evaluate the risk of bleeding. Combining the comprehension of these parameters with a more refined image of how and where platelets are born might help find the cause and best therapy when platelet counts are low.

\section{Keywords}

Platelets - Immune thrombocytopenia . Megakaryocytes · Thrombopoiesis · Bone marrow 
Zusammen mit unseren Daten aus der Intravitalmikroskopie kann somit das „Flow-Modell“ modifiziert werden: Reife MKs an der Gefäßnische im Knochenmark geben größere zytoplasmatische Fragmente (Präplättchen) in den Blutstrom ab, wobei so der vorzeitige prothrombotische Kontakt mit dem Subendothel minimiert werden kann. Der letzte Schritt der Plättchenbiogenese erfolgt dann über Proplättchenzwischenformen auf dem Weg zur Lunge bzw. im Kapillarbett der Lunge. Dies erklärt zudem, warum nur sehr wenige Reste von MKs (nackte Kerne) im roten Knochenmark detektierbar sind [26]. Obwohl einige Puzzleteile damit schon gut ineinanderpassen, sind weitere Experimente notwendig, um zu einem konsistenten Bild der Thrombozytopoese zu kommen, das alle bisherigen In-vitro- und In-vivo-Daten mit einschließt. Dies ist auch wichtig, um die pathologischen Veränderungen besser zu verstehen, wie die Bildung von Autoantikörpern gegen MKs oder gegen Thrombozyten, wie z. B. bei der Immunthrombozytopenie (ITP).

\section{Implikationen für die Diagnose von Thrombozytopenien}

Thrombozytopenien können vielfältige Ursachen zugrunde liegen, wie z. B. gesteigerter Umsatz von Thrombozyten (z. B. ITP), verminderte Thrombozytenbildung (z. B. nach myeloablativer Therapie) oder assoziiert mit Thrombozytenfunktionsstörungen (z. B. bei $\mathrm{MYH}$-assoziierten Thrombozytopenien). Die besten Therapieoptionen bei Thrombozytopenie können natürlich erst dann gewählt werden, wenn ihre Ursache bekannt ist. So ist bei Kindern nach myeloablativer Therapie das Blutungsrisiko deutlich höher als bei der ITP - trotz gleich erniedrigter Thrombozytenzahl. Diese „nackte“ Thrombozytenzahl, wie sie zunächst in automatisierten Blutzählgeräten bestimmt wird, ist daher nur bedingt in der Lage, ein Blutungsrisiko vorauszusagen bzw. jener „Ariadne-Faden“, der den Arzt durch den Irrgarten thrombozytopenischer Erkrankungen weist, wie es sich der deutsche Internist Erich Frank (Breslau, ab 1933 Istanbul) bereits 1925 gewünscht hat [10].
Zusätzliche Plättchenparameter, die über Größe, Granularität, Populationsverteilung usw. Auskunft geben, können in Zukunft bei der Differenzialdiagnose helfen - zumal Knochenmarkbiopsien oft nicht vorliegen, die Auskunft über Anzahl und Morphologie der MKs geben könnten.

Einige dieser Parameter seien hier vorgestellt:

- MPV („Mean Platelet Volume“): gibt das durchschnittliche Volumen eines Thrombozyten an und ist analog zum mittleren korpuskulären Volumen (MCV) bei den Erythrozyten. Typische Werte sind 9-12 fl. Erniedrigte Werte findet man beim Wiskott-Aldrich-Syndrom, erhöhte Werte beim Bernard-Soulier-Syndrom oder bei den Makrothrombozytopenien mit Mutationen im $\mathrm{MYH}_{9}$-Gen.

- PDW („Platelet Distrubution Width“): gibt Information über die Homogenität der Thrombozytenpopulation und entspricht dem RDW („Red Blood Cell Distribution Width“). So finden sich z. B. bei reaktiver Thrombozytenbildung aufgrund sekundärer Thrombozytose größere Plättchen im Blut, die das MPV kaum beeinflussen, aber einen Effekt auf das PDW haben [22]. Unglücklicherweise wird in einigen Zählgeräten PDW als Verteilungsweite bei 20\% des MaximalPeaks der Thrombozytenverteilungskurve (in fl) definiert, während andere Automaten einen prozentualen PDW berechnen, als Standardabweichung der einzelnen Thrombozytenvolumina, geteilt durch das MPV.

- P-LCR („Platelet Large Cell Ratio“): wird berechnet als die Fraktion von Thrombozyten, die größer als $12 \mathrm{fl}$ sind und kleiner als der obere Plättchendiskriminator (oft $20 \mathrm{fl}$ ) in Bezug zur Gesamtzahl. Thrombozytenwerte können falsch-niedrig sein, wenn sehr große Thrombozyten nicht gezählt werden, oder falsch-niedrig, wenn kleine Retikulozyten im Thrombozytenfenster detektiert werden. P-LCR könnte dazu beitragen, Patienten mit jungen, großen Thrombozyten zu identifizieren.

- PCT (Plättchenkrit): wurde in Analogie zum Hämatokrit entwickelt und dient als Maß für die Plättchenmasse. Er wird errechnet als Produkt von MPV und der Thrombozytenzahl, wobei der Referenzwert bei etwa 0,25\% liegt. Obwohl die klinische Relevanz noch bestimmt werden muss, legen Studien an Tieren nahe, dass der Plättchenkrit besser als die bloße Thrombozytenzahl geeignet ist, um eine Thrombozytopenie zu definieren [32].

- IPF („Immature Platelet Fraction“): ist sicherlich der Parameter mit dem größten diagnostischen Potenzial in der Zukunft. Die Messung erfolgt optisch und setzt die Zugabe von Fluoreszenzfarbstoffen, wie sie für die Messung der Retikulozyten verwendet wird, voraus. Da junge Thrombozyten etwas größer sind als alte und zudem noch Reste an mRNA aufweisen, die mit dem Farbstoff angefärbt werden können, kann so die Fraktion an großen, RNA-reichen Thrombozyten bestimmt werden. Diese Thrombozyten weisen morphologisch eine netzartige Anfärbbarkeit auf und werden auch als „,retikuliert" bezeichnet. Obwohl sie nachweislich hämostatisch aktiver sind als normale Thrombozyten, werden sie als ,immatur“ bezeichnet, was sicherlich eine Fehlbenennung ist. Erhöhte IPF-Werte finden sich bei verstärktem Umsatz der Thrombozyten (z. B. bei der ITP), während die Werte bei angeborenen Thrombozytopenien oder nach myeloablativer Therapie im Normbereich bleiben [5]. Bei zunehmender Kenntnis des Zusammenhangs zwischen ITP und Megakaryozytopoese könnte der IPF-Wert in Zukunft zumindest einen Teil der Knochenmarkbiopsien ersetzen und damit so etwas wie ein Thrombozyten-Bildungs-Index entwickelt werden. Während IPF prozentual bestimmt wird, kann durch Multiplikation mit der Thrombozytenzahl auch ein absoluter IPF-Wert errechnet werden, der in einer präliminären Studie unserer Gruppe auch differenzialdiagnostisch informativ ist (Manuskript unter Revision). 


\section{Fazit für die Praxis}

Thrombozyten entstehen als zytoplasmatische Abschnürungen von Megakaryozyten, die im Knochenmark Thrombozyten in die Blutbahn abgeben. Dabei werden zunächst größere Fragmente freigesetzt, die im Blutstrom bzw. bei der Lungenpassage zu reifen Thrombozyten differenzieren. Der Anteil von größeren, jüngeren Thrombozyten kann gemessen werden, wobei die Aussagekraft der zusätzlichen Thrombozytenparameter zur Differenzialdiagnose von Thrombozytopenien noch weiter evaluiert werden muss. Die Herausforderung für die kommenden Jahre besteht darin, diese $\mathrm{Pa}$ rameter für den klinischen Alltag in Anwendung zu bringen. Dazu gehört neben der Diagnostik auch das prognostische Potenzial, um z. B. zu bestimmen, wann nach Rekonstitution des Knochenmarks ein Thrombozyten-Engraftment stattgefunden hat. Es wird sicherlich noch eine Weile dauern, ehe patientenspezifische Thrombozyten in ausreichender Zahl in vitro hergestellt werden können. Die kleinste aller Blutzellen verbirgt noch stets das Geheimnis seiner Geburt.

\section{Korrespondenzadresse \\ PD Dr. H. Schulze}

Labor für Pädiatrische Molekularbiologie, Klinik für Allgemeine Pädiatrie, Otto-Heubner Centrum für Kinder- und Jugendmedizin Ziegelstr. 5-9, 10098 Berlin harald.schulze@charite.de

Danksagung. Der Autor dankt Prof. Dr. Gerhard Gaedicke für Anmerkungen zum Artikel.

Interessenkonflikt. Der korrespondierende Autor gibt an, dass kein Interessenkonflikt besteht.

\section{Literatur}

1. Aschoff L (1893) Über capilläre Embolie riesenkernhaltiger Zellen. Arch Path Anat Physiol 134: 11

2. Avecilla ST, Hattori K, Heissig B et al (2004) Chemokine-mediated interaction of hematopoietic progenitors with the bone marrow vascular niche is required for thrombopoiesis. Nat Med 10:64-71

3. Battinelli EM, Hartwig JH, Italiano JE Jr (2007) Delivering new insight into the biology of megakaryopoiesis and thrombopoiesis. Curr Opin Hematol 14:419-426

4. Behnke O, Forer A (1998) From megakaryocytes to platelets: platelet morphogenesis takes place in the bloodstream. Eur J Haematol Suppl 61:3-23
5. Briggs C, Kunka S, Hart D et al (2004) Assessment of an immature platelet fraction (IPF) in peripheral thrombocytopenia. Br J Haematol 126:93-99

6. Chang Y, Bluteau D, Debili N, Vainchenker W (2007) From hematopoietic stem cells to platelets. J Thromb Haemost 5 (Suppl 1):318-327

7. Choi ES, Nichol JL, Hokom MM et al (1995) Platelets generated in vitro from proplatelet-displaying human megakaryocytes are functional. Blood 85:402-413

8. De Botton S, Sabri S, Daugas E et al (2002) Platelet formation is the consequence of caspase activation within megakaryocytes. Blood 100:1310-1317

9. Deutsch VR, Tomer A (2006) Megakaryocyte development and platelet production. Br J Haematol 134:453-466

10. Frank E (1925) Hämorrhagische Diathesen. In: Schittenhelm A (Hrsg) Handbuch der Krankheiten des Blutes und der blutbildenden Organe. Springer, Berlin

11. Hartwig JH (2006) The platelet: form and function. Semin Hematol 43:S94-S100

12. Howell WH, Donahue DD (1937) The production of blood platelets in the lung. J Exp Med 65:177

13. Italiano JE Jr, Lecine P, Shivdasani RA, Hartwig JH (1999) Blood platelets are assembled principally at the ends of proplatelet processes produced by differentiated megakaryocytes. J Cell Biol 147:12991312

14. Junt T, Schulze H, Chen Z et al (2007) Dynamic visualization of thrombopoiesis within bone marrow. Science 317:1767-1770

15. Kaufman RM, Airo R, Pollack S, Crosby WH (1965) Circulating megakaryocytes and platelet release in the lung. Blood 26:720-731

16. Kaushansky K (1995) Thrombopoietin: the primary regulator of platelet production. Blood 86:419431

17. Kaushansky K (2008) Historical review: megakaryopoiesis and thrombopoiesis. Blood 111:981-986

18. Kosaki G (2008) Platelet production by megakaryocytes: protoplatelet theory justifies cytoplasmic fragmentation model. Int J Hematol 88:255-267

19. Larson MK, Watson SP (2006) A product of their environment: do megakaryocytes rely on extracellular cues for proplatelet formation? Platelets 17:435-440

20. Lecine P, Villeval JL, Vyas P et al (1998) Mice lacking transcription factor NF-E2 provide in vivo validation of the proplatelet model of thrombocytopoiesis and show a platelet production defect that is intrinsic to megakaryocytes. Blood 92:1608-1616

21. Lichtman MA, Chamberlain JK, Simon W, Santillo PA (1978) Parasinusoidal location of megakaryocytes in marrow: a determinant of platelet release. Am J Hematol 4:303-312

22. Osselaer JC, Jamart J, Scheiff JM (1997) Platelet distribution width for differential diagnosis of thrombocytosis. Clin Chem 43:1072-1076

23. Patel SR, Hartwig JH, Italiano JE Jr (2005) The biogenesis of platelets from megakaryocyte proplatelets. J Clin Invest 115:3348-3354

24. Patel-Hett S, Richardson JL, Schulze H et al (2008) Visualization of microtubule growth in living platelets reveals a dynamic marginal band with multiple microtubules. Blood 111:4605-4616

25. Pedersen NT (1971) Circulating megakaryocytes in blood from the inferior vena cava in adult rats. Scand J Haematol 8:223-230

26. Pedersen NT (1978) Occurrence of megakaryocytes in various vessels and their retention in the pulmonary capillaries in man. Scand J Haematol 21:369-375
27. Radley JM, Haller CJ (1982) The demarcation membrane system of the megakaryocyte: a misnomer? Blood 60:213-219

28. Richardson JL, Shivdasani RA, Boers C et al (2005) Mechanisms of organelle transport and capture along proplatelets during platelet production. Blood 106:4066-4075

29. Schulze H, Dose M, Korpal M et al (2008) RanBP10 is a cytoplasmic guanine nucleotide exchange factor that modulates noncentrosomal microtubules. J Biol Chem 283:14109-14119

30. Schulze H, Korpal M, Hurov J et al (2006) Characterization of the megakaryocyte demarcation membrane system and its role in thrombopoiesis. Blood 107:3868-3875

31. Schwertz H, Koster S, Kahr WH et al (2010) Anucleate platelets generate progeny. Blood 115:38013809

32. Tvedten $\mathrm{H}$, Lilliehook I, Hillstrom A, Haggstrom J (2008) Plateletcrit is superior to platelet count for assessing platelet status in Cavalier King Charles Spaniels. Vet Clin Pathol 37:266-271

33. Wright J (1906) The origin and nature of blood platelets. Boston Med Surg J 154:643-645

34. Yamada $E$ (1957) The fine structure of the megakaryocyte in the mouse spleen. Acta Anat (Basel) 29:267-290 